\title{
Recovery and validation of Mars ionospheric electron density profiles from Mariner 9
}

\author{
Paul Withers ${ }^{1,2^{*}}$, Sarah Weiner ${ }^{1}$ and Nicholas Roy Ferreri ${ }^{1}$
}

\begin{abstract}
Electron density profiles from the ionosphere of Mars that were obtained by the Mariner 9 radio occultation experiment in 1971-1972 have unique scientific value because they extend to higher altitudes than comparable datasets and were acquired during a tremendous dust storm that had substantial and poorly understood effects on the ionosphere. Yet these profiles are not publicly available in an accessible format. Here, we describe the recovery of these profiles, which are made available as part of this article. The validity of the profiles was tested by using them to explore the effects of a dust storm on the topside ionosphere, the morphology of the topside ionosphere, the behavior of the M1 layer, and possible meteoric layers. The dust storm that waned over the course of the primary mission (November-December 1971) had major effects on the ionosphere of Mars. It elevated the M1 and M2 layers of the ionosphere by $20-30 \mathrm{~km}$, but the separation of the two layers stayed fixed throughout the primary mission, which suggests that the neutral atmosphere at these altitudes was not heated during the dust storm. However, the altitude of the $1500 \mathrm{~cm}^{-3}$ density level, a proxy for the top of the ionosphere, decreased steadily by $74 \pm 12 \mathrm{~km}$ over the course of the primary mission. Mariner 9 observations of the topside ionosphere differ from comparable Mars Express observations. Compared to Mars Express, the Mariner 9 data, which were acquired during a period of relatively high solar wind dynamic pressure, have lower densities at high altitudes. They are also more likely to have a "one scale height" morphology than a "two scale height" morphology. The peak density of the M1 layer depends on solar zenith angle and solar irradiance similarly to previous studies with Mars Global Surveyor observations, which indicates that dust storms do not affect the behavior of the peak density. No clear meteoric layers were identified.
\end{abstract}

Keywords: Mars, lonosphere, Mariner 9

\section{Introduction}

Mariner 9, the first spacecraft to orbit another planet, reached Mars on 13 November 1971 and settled into a 12$\mathrm{h}$ orbit with an inclination of $65^{\circ}$ (Kliore et al. 1972a, b). It conducted radio occultation measurements of the atmosphere and ionosphere of Mars in November-December 1971 (primary mission) and May-June 1972 (extended mission) (Kliore et al. 1970, 1973). These observations discovered the immense topographic range of the planet $(30 \mathrm{~km})$, the strong response of the atmosphere and its embedded ionosphere to suspended dust, and the dayto-day stability of the ionosphere (Cain et al. 1972, 1973; Kliore 1973). Over the course of its mission, Mariner

\footnotetext{
*Correspondence: withers@bu.edu

1 Department of Astronomy, Boston University, 725 Commonwealth Avenue, Boston, MA 02215, USA

${ }^{2}$ Center for Space Physics, Boston University, 725 Commonwealth Avenue, Boston, MA 02215, USA
}

9 acquired several hundred radio occultation measurements. In this technique, a radio link between the spacecraft and Earth is monitored as the spacecraft goes behind Mars as seen from Earth (ingress) and as the spacecraft emerges from behind Mars as seen from Earth a short time later (egress) (Withers 2009a). However, the mission was only able to obtain viable ionospheric electron density profiles for ingress occultations of the dayside, about 114 profiles. Egress opportunities relied on the stability of the onboard radio oscillator, which was sufficient for the detection of the strong signature of the neutral atmosphere, but not the weaker signature of the ionosphere, whereas ingress opportunities could use a two-way technique stabilized by a higher-performing Earth-based oscillator that was able to measure both the ionosphere and neutral atmosphere. Nightside occultations rarely produced robust electron density profiles due to low nightside densities.

\section{包 Springer}

(C) 2016 Withers et al. Open Access This article is distributed under the terms of the Creative Commons Attribution 4.0 International License (http://creativecommons.org/licenses/by/4.0/), which permits unrestricted use, distribution, and reproduction in any medium, provided you give appropriate credit to the original author(s) and the source, provide a link to the Creative Commons license, and indicate if changes were made. 
Previous analyses of the Mariner 9 electron density profiles have focused on the behaviors of the topside ionosphere and the main peak of the ionosphere. In the topside, they have interpreted the lack of distinctive ionopause features at the top of the ionosphere in terms of pressure balance with the solar wind (Kliore 1992; Luhmann 1992; Luhmann et al. 1990; Shinagawa 1996; Slavin and Holzer 1982; Zhang and Luhmann 1992). They have noted that the morphology of the topside ionosphere can be described by an exponential decay in electron density with altitude, where the relevant scale height is uniform with altitude (Breus et al. 1998; Kliore 1992; Ness et al. 2000; Shinagawa 1996; Slavin and Holzer 1982). They have shown that the topside plasma scale height increases with increasing solar irradiance (Bauer and Hantsch 1989; Fjeldbo et al. 1977; Ness et al. 2000; Shinagawa 1996; Stewart and Hanson 1982). They have concluded that the topside plasma scale height is not proportional to the plasma temperature (Zhang et al. 1990). They have inferred the presence of a horizontal magnetic field in the ionosphere from the fact that the morphologies of vertical profiles are photochemical equilibrium-like up to $240 \mathrm{~km}$ (Breus et al. 1998; Luhmann et al. 1990; Ness et al. 2000).

At the main ionospheric peak, they have found that the peak electron density is proportional to the square root of the cosine of solar zenith angle (Hantsch and Bauer 1990; Kliore 1992; Kliore et al. 1973; Luhmann and Brace 1991; Schunk and Nagy 1980; Stewart and Hanson 1982; Zhang et al. 1990). They have found, with the support of additional datasets, that the peak electron density increases with increasing solar irradiance (Hantsch and Bauer 1990; Shinagawa 1996; Stewart and Hanson 1982). They have found, with the support of additional datasets, that the altitude of the peak electron density increases with increasing solar zenith angle (Hantsch and Bauer 1990; Luhmann and Brace 1991; Shinagawa 1996; Stewart and Hanson 1982; Zhang et al. 1990). They have discovered that the ionospheric peak altitude is elevated significantly during dust storms (Hantsch and Bauer 1990; Kliore et al. 1973; Luhmann and Brace 1991; Shinagawa 1996; Stewart and Hanson 1982; Wang and Nielsen 2003; Withers and Pratt 2013; Zhang et al. 1990).

The Mariner 9 electron density profiles have been published graphically in Kliore et al. (1972a, b, 1973), Zhang et al. (1990), and Kliore (1992). They were archived as microfilmed data tables and images as dataset PSPA-00141 at the National Space Science Data Center (NSSDC), and this archive is described further at http://nssdc.gsfc.nasa.gov/nmc/datasetDisplay. do?id=PSPA-00141. However, they have never been made readily available in digital format. Although some senior scientists may have retained copies of this dataset over four decades and many generations of computer equipment, the vast majority of active scientists do not have access to these profiles. The latter are the intended readers of this article.

Relative to the Mars Global Surveyor (MGS) dataset (Hinson 2007), which contains the only electron density profiles for Mars that are readily available to scientists today, the Mariner 9 dataset has some unique characteristics:

1. High vertical extent. The Mariner 9 profiles routinely extend above $300 \mathrm{~km}$, with many approaching 400 $\mathrm{km}$, whereas MGS profiles rarely exceed $200 \mathrm{~km}$ (Hinson 2007). The typical boundary between ionospheric and solar wind plasma is $400 \mathrm{~km}$, which is sometimes called the ionopause or photoelectron boundary (Mitchell et al. 2001).

2. Measurements during a tremendous dust storm. Mariner 9's first images from orbit, acquired as an unsurpassed dust storm was raging, showed nothing but dust and the Tharsis volcanic peaks. Conditions in the atmosphere and ionosphere were severely affected by this storm. The ionospheric peak was 20 $30 \mathrm{~km}$ higher during this storm than normal (Hantsch and Bauer 1990), indicating immense expansion of the lower atmosphere due to suspended dust.

3. Global coverage. The Mariner 9 profiles are globally distributed, whereas almost all of the 5600 MGS profiles are north of $60^{\circ} \mathrm{N}$ (Hinson 2007). The only ones that are not are 220 profiles between $70^{\circ} \mathrm{S}$ and $64^{\circ} \mathrm{S}$. The Mariner 9 profiles permit exploration of the ionosphere within the tropical neutral atmosphere and above a range of crustal magnetization conditions (Acuña et al. 2001).

4. Solar zenith angle (SZA) coverage. SZA is the major factor controlling ionospheric conditions on Mars (Withers 2009b). All MGS profiles have SZAs of $71^{\circ}$ or more, where the Sun is very low on the horizon (Hinson 2007). By contrast, the Mariner 9 profiles sample SZAs as low as $47^{\circ}$, closer to the subsolar point, which causes higher electron densities.

The aims of this article are to make the recovered Mariner 9 electron density profiles widely available, to describe how these profiles were recovered from the NSSDC microfilm, and to place these profiles in the context of current ionospheric studies. To address the last of these aims, the profiles were analyzed. Our analysis focused on topics that had not previously been addressed using these profiles, namely the effects of a dust storm on the topside ionosphere, the morphology of the topside ionosphere, the behavior of the M1 layer, and possible meteoric layers. The M1 layer is visible in electron density profiles as a shoulder approximately $25-30 \mathrm{~km}$ below the main M2 layer (Fallows et al. 2015). As these topics have recently been addressed using other datasets, the analysis 
reported here helps to place the recovered Mariner 9 electron density profiles in the context of current ionospheric studies. It also serves to validate the recovered profiles.

The section "Recovery of Mariner 9 electron density profiles" describes the recovery of the Mariner 9 electron density profiles. The section "Scientific analysis" presents the results of our scientific analysis, with the section "Effects of a dust storm on the topside ionosphere" addressing the top of the ionosphere, the section "Vertical structure of the topside ionosphere" addressing the vertical structure of the topside ionosphere, the section "M1 peak electron density and solar zenith angle" addressing the relationship between M1 peak density and solar zenith angle, the section "M1 peak electron density and solar irradiance" addressing the relationship between M1 peak density and solar irradiance, the section "Separation between the M1 and M2 layers" addressing the separation between the M1 and M2 layers, and the section "Possible meteoric layers" addressing possible meteoric layers. The section "Discussion and conclusions" summarizes the conclusions of this work.

\section{Recovery of Mariner 9 electron density profiles}

Upon response to an initial inquiry concerning access to the dataset PSPA-00141, NSSDC staff provided us with images of 25 sample pages from Rev 57 (revolution or orbit 57), which occurred on the day of the year 346 of 1971 (12 December). These included data tables for the ingress occultation showing electron density, plasma scale height, and inferred plasma temperature as functions of radial distance and height; neutral mass density, number density, temperature lapse rate, and pressure scale height as functions of radial distance and height; and ellipsoid parameters. Plots of the ingress results and tables of neutral data products for the egress occultation were also included.

Based on these samples, we requested data tables and plots of ionospheric properties as functions of radial distance for all occultations for which ionospheric peak properties were reported in Kliore et al. (1972b) and Kliore et al. (1973). We assumed that the absence of reported peak properties for a given occultation meant that no useful ionospheric profile was delivered to the archive. This approach primarily selected ingress occultations with solar zenith angles less than $90^{\circ}$, although a handful of the selected occultations have solar zenith angles between $90^{\circ}$ and $100^{\circ}$. Neutral properties, although present in the archive and available to other interested researchers, were not deemed useful for our purposes.

We provided a list of selected orbits to the NSSDC and subsequently received approximately 768 TIFF images of microfilmed information. A typical image is shown in Fig. 1. The information in these images of data tables was processed using optical character recognition software, incorrectly recognized digits (which were legion) were manually corrected, and results were stored as ASCII tables. Quality control for each occultation was ensured by reproducing the accompanying electron density-radial distance plot and any similar plots in prior publications (Kliore 1992; Kliore et al. 1972a, b, 1973; Zhang et al. 1990). This process generated an electron density-radius profile labeled by its orbit number for each available ingress occultation.

For productive scientific analysis, additional supporting information is required. Tables in Kliore et al. (1972b) and Kliore et al. (1973) report latitude, longitude, solar zenith angle, peak electron density, peak radial distance, and peak altitude for each occultation. We verified that the peak electron densities and peak radial distances reported by Kliore et al. (1972b) and Kliore et al. (1973) are consistent with the recovered electron density profiles.

Martian geodesy has evolved considerably since the Mariner era, so the published latitudes, longitudes, and peak altitudes need careful consideration. Generally speaking, pre-MGS work tended to use areographic latitudes and post-MGS work has tended to use areocentric latitudes. However, Kliore et al. (1973) define latitude in terms of a spherical planet, which leads to areocentric latitudes. Since the difference between latitudes defined with these two conventions is in any case always less than $1^{\circ}$, we have interpreted the reported latitudes as modernera areocentric values (Smith et al. 2001). Longitudes in Kliore (1972b, 1973) are west longitudes, which were converted into east longitudes by subtracting them from $360^{\circ}$. Tracking the many different reference areoids used over the past four decades to convert radial distance to altitude is a major undertaking, which we sidestepped by converting each occultation's list of radial distances into altitudes using the radius of the MOLA areoid at the appropriate latitude and longitude (Smith et al. 2001).

Precise timing information is not readily available for these profiles, although it could perhaps be provided by a diligent investigation of the relevant SPICE kernels (http://naif.jpl.nasa.gov/pub/naif/M9/kernels/). We assigned dates to each occultation based on the day of the year information written on the microfilmed tables.

The end product is a set of 114 electron density profiles as functions of radial distance and altitude, each accompanied by orbit number, date, latitude, longitude, solar zenith angle, radius of areoid, radial distance to maximum electron density, altitude of maximum density, and maximum electron density. This dataset accompanies this manuscript as Additional file 1 and is also in the process of being archived at the NASA Planetary Data System (PDS). At the time of writing, the dataset is under review and is available at http:// ppi.pds.nasa.gov/archive1/MR9RSS_0001/.Electron density uncertainties were neither archived nor discussed 


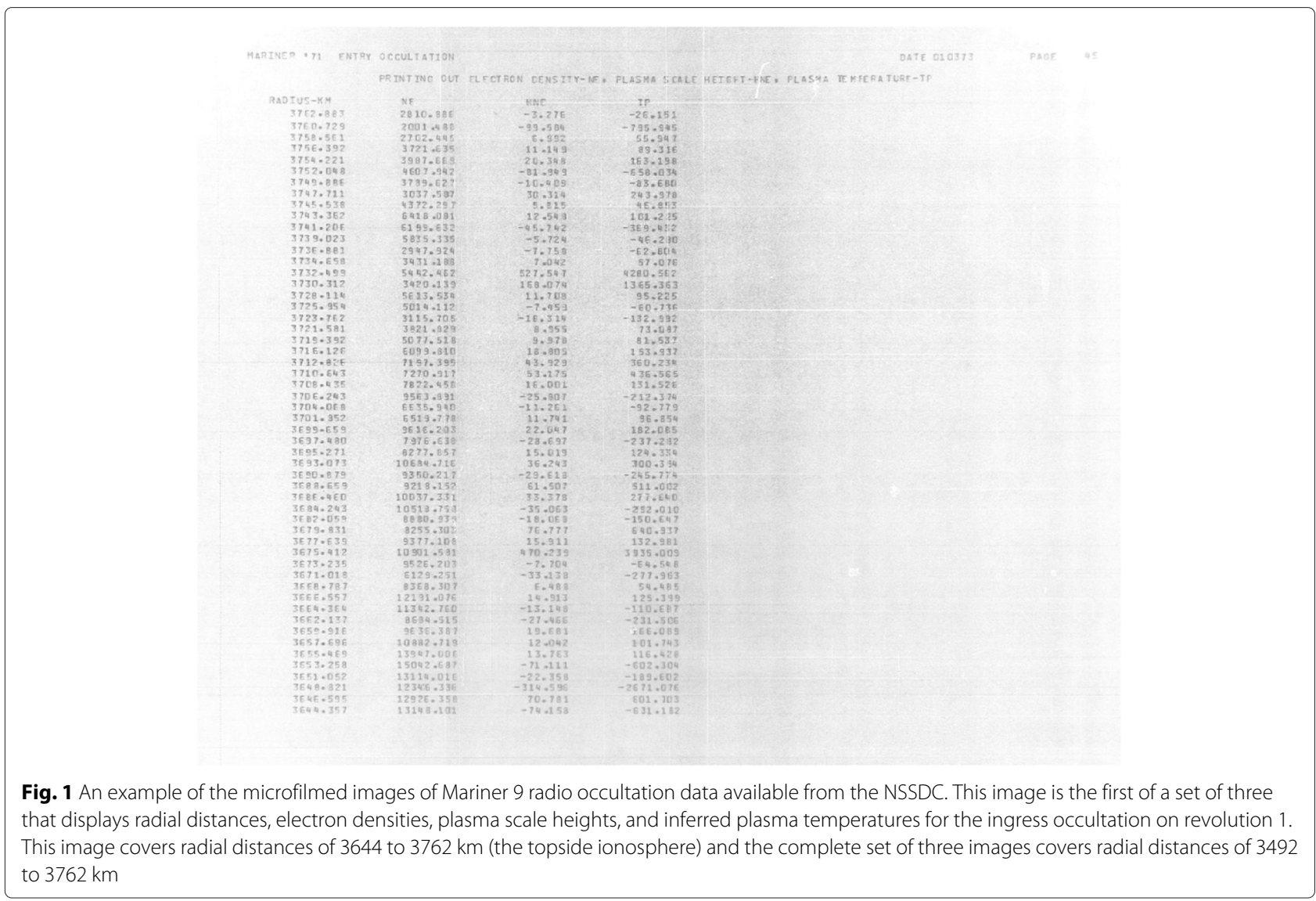

extensively in publications from the Mariner 9 era, but they can be estimated for each profile as the smallest value in that profile.

Seventy-eight profiles were acquired during the primary mission on orbits 1-79 (solar zenith angles of $47^{\circ}-$ $57^{\circ}$ ) and 36 profiles were acquired during the extended mission on orbits $352-450$ (solar zenith angles of $72^{\circ}-$ $99^{\circ}$ ). In the primary mission, profiles typically spanned 80 to $300 \mathrm{~km}$ with a vertical resolution of $2 \mathrm{~km}$ and a lowest reported density of $700 \mathrm{~cm}^{-3}$. In the extended mission, profiles typically spanned 80 to $240 \mathrm{~km}$ with a vertical resolution of $1 \mathrm{~km}$ and a lowest reported density of $1200 \mathrm{~cm}^{-3}$. Point-to-point fluctuations in electron density are noticeably greater in the profiles from the extended mission than in those of the primary mission. The high-gain antenna was not pointed at Earth during extended mission occultations, which reduced the signal-to-noise ratio of the experiments (Kliore et al. 1973). Consequently, we focus exclusively on the primary mission profiles in several of the analyses reported below.

The geographic coverage of the Mariner 9 dataset is shown in Fig. 2. Figure 3 compares a plot of seven early profiles from the recovered dataset to a similar plot from an original publication; the agreement is excellent.
The profile from orbit $4\left(39.1^{\circ} \mathrm{S}, 209.9^{\circ} \mathrm{E}\right)$ is quite unusual: its peak density is more than $30 \%$ smaller than the peak density of any other occultation from the primary mission and its highest altitude data point is $60 \mathrm{~km}$ below those of any other occultation from the first 50 orbits. This may be connected with the unique nature of the local

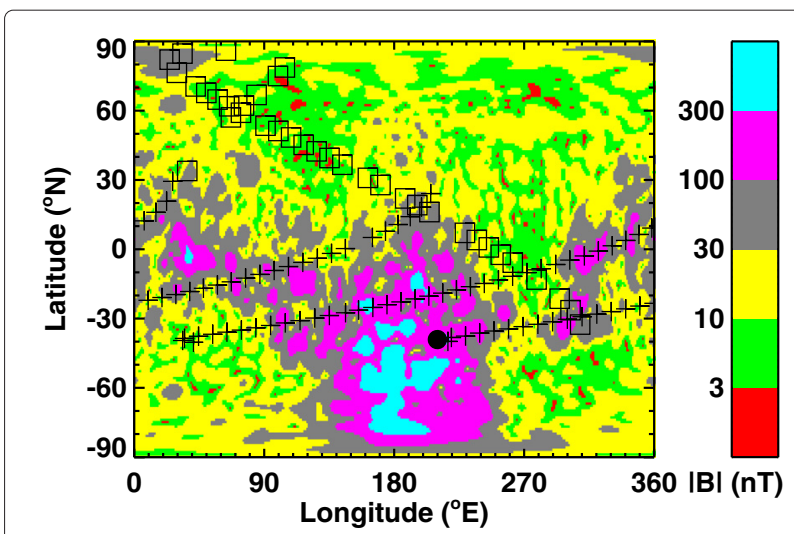

Fig. 2 Geographical coverage of the Mariner 9 electron density profiles. Crosses and squares indicate occultations from the primary and extended missions, respectively. The filled circle at $39.1^{\circ} \mathrm{S}, 209.9^{\circ} \mathrm{E}$ highlights orbit 4. Colors indicate the magnetic field strength at 150 km based upon the model of Arkani-Hamed (2004) 


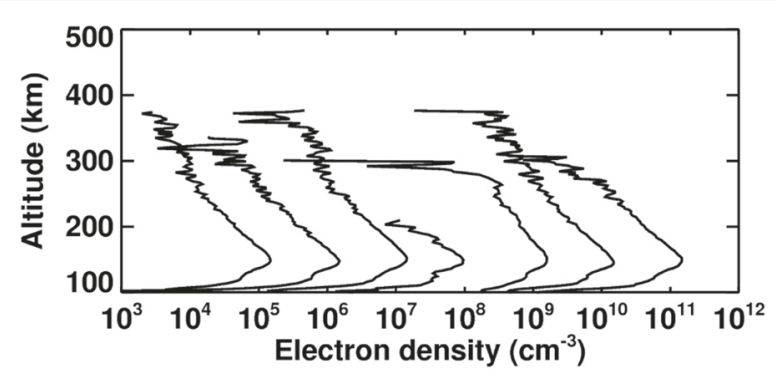

MARINER 9 REVS 1-7 ENTRY

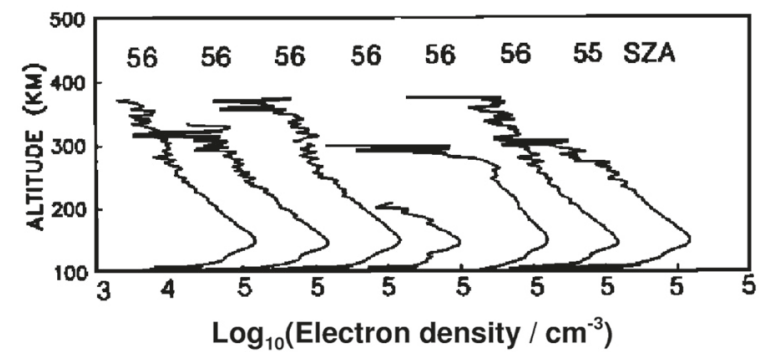

Fig. 3 Top. Electron density profiles from ingress occultations on orbits $1-7$, based on the data recovered in this project. Electron densities in the profiles from orbits 1, 2, 3, 4, 5, 6, and 7 have been multiplied by factors of $10^{0}, 10^{1}, 10^{2}, 10^{3}, 10^{4}, 10^{5}$, and $10^{6}$, respectively, for clarity. This panel is truncated at $100 \mathrm{~km}$ for consistency with the bottom panel. Bottom. Electron density profiles from ingress occultations on orbits 1-7, as reported by Zhang et al. (1990). A similar scaling was also applied to the electron density profiles in this panel. Numbers above each profile indicate their solar zenith angles (degrees). Bottom panel reproduced from Fig. 7 of Zhang et al. (1990) by permission of American Geophysical Union

magnetic field conditions. The magnetic field strength at $150 \mathrm{~km}$ at the location of this occultation is $270 \mathrm{nT}$ (Arkani-Hamed 2004), and the frequency of occurrence of open magnetic field lines on the dayside at this location exceeds $40 \%$ (Brain et al. 2007). None of the other Mariner 9 occultations have both a magnetic field strength above $210 \mathrm{nT}$ and an open field line occurrence of over $33 \%$. This unusual profile contrasts markedly with the results of Nielsen et al. (2007), who reported several case studies in which peak densities were approximately doubled where the magnetic field is strong and vertical.

\section{Scientific analysis}

\section{Effects of a dust storm on the topside ionosphere}

The ionospheric peak was $20-30 \mathrm{~km}$ higher than normal during the Mariner 9 primary mission (Hantsch and Bauer 1990), indicating immense expansion of the lower atmosphere due to suspended dust during this tremendous dust storm. This unusual behavior was immediately noted by the early investigators, who attributed it to the subsidence of the neutral atmosphere during the waning phase of a tremendous dust storm (Kliore et al. 1973; McElroy 1977). The steady descent of the ionospheric peak during the primary mission of Mariner 9 becomes noticeably clearer when peak altitudes are updated from being referenced to the Mariner-era areoid (Kliore et al. $1972 \mathrm{~b}$ ) to being referenced to the MOLA areoid (Withers and Pratt 2013). Withers and Pratt (2013) showed how peak altitude dropped steadily from 150 to $140 \mathrm{~km}$ from orbit 1 to 79 . This cannot be attributed to solar zenith angle effects. They concluded that "upper atmospheric densities were 12 times greater than usual at the time of Mariner 9's arrival at Mars. Even larger densities likely occurred at earlier times during this remarkable storm". The ionospheric peak has also been observed to change during other dust events (e.g., Bougher et al. 2001; Wang and Nielsen 2003; Withers and Pratt 2013). However, the effects of a dust storm on the topside ionosphere have not been addressed previously. We focus on the altitude of the top of the ionosphere.

We define the top of the ionosphere as the altitude above the peak at which electron densities first fall below 1500 $\mathrm{cm}^{-3}$ when moving upward in altitude. This threshold value of $1500 \mathrm{~cm}^{-3}$ is larger than used in comparable work (Kliore 1992), but the adoption of a smaller value would lead to non-detections of the top of the ionosphere in too many profiles. Here, we are primarily interested in relative trends in the altitude of the top of the ionosphere, not its absolute value. Figure 4 shows how the altitude of the top of the ionosphere varies with solar zenith angle. Also shown are similar altitudes from Viking Orbiter occultations, which were manually extracted from figures in Zhang et al. (1990). For profiles in which the top of the ionosphere was not detected, the altitude of the top of the ionosphere is assumed to be the altitude of the highest data point in the profile. There is no appreciable difference between altitudes inferred through such non-detections and altitudes found by actual detections.

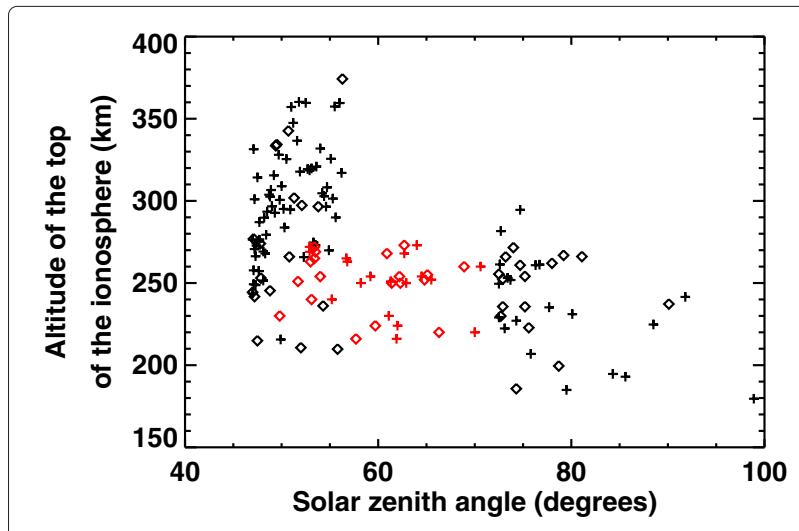

Fig. 4 Altitude of the top of the ionosphere, as defined in the text, as a function of solar zenith angle. Black and red symbols indicate Mariner 9 and Viking Orbiter occultations, respectively. Crosses indicate actual detections of the top of the ionosphere, and diamonds indicate lower limits from non-detections. Orbit 4's result is a non-detection of $210 \mathrm{~km}$ at a solar zenith angle of $55.8^{\circ}$ 
Relative to the Mariner 9 extended mission and the Viking mission, the top of the ionosphere extended to unusually high altitudes during the Mariner 9 primary mission. The possible influence of solar zenith angle effects is excluded by results from the Mariner 9 primary mission data being considerably higher than Viking results at the same solar zenith angle. The unusually high altitudes appear to be connected with the waning dust storm, as the top of the ionosphere descends as the orbit number increases (Fig. 5). The correlation coefficient is -0.58 . A linear fit to the data points gives an initial altitude of $330 \pm 7 \mathrm{~km}$ and a decrease in altitude of $0.94 \pm 0.15 \mathrm{~km}$ per orbit, or a decrease in the altitude of the top of the ionosphere by $74 \pm 12 \mathrm{~km}$ from orbit 1 to orbit 79, a period of 39 days.

The systematic nature of this trend argues against it being caused by variations in solar conditions or the magnetic environment. It is reasonable that the expansion of the neutral atmosphere during the dust storm can elevate the top of the ionosphere much as the ionospheric peak is elevated. However, the magnitudes of the altitude changes are very different. Over the course of the primary mission, the ionospheric peak descends by about $10 \mathrm{~km}$, yet the top of the ionosphere descends by about $70 \mathrm{~km}-$ seven times more. This is illustrated in Fig. 6 by a representative set of profiles.

For data from the Mariner 9 extended mission and the Viking orbiters, the top of the ionosphere occurs at approximately $250 \mathrm{~km}$ altitude. That is, electron densities above $250 \mathrm{~km}$ are less than $1500 \mathrm{~cm}^{-3}$. This differs from the work of Duru et al. (2008), who used in situ measurements of local electron densities collected by the MARSIS instrument on Mars Express (MEX) in 2005-2007 to find that electron densities at $300-350 \mathrm{~km}$ and solar zenith angles less than $80^{\circ}$ are on the order of $2000-4000 \mathrm{~cm}^{-3}$.

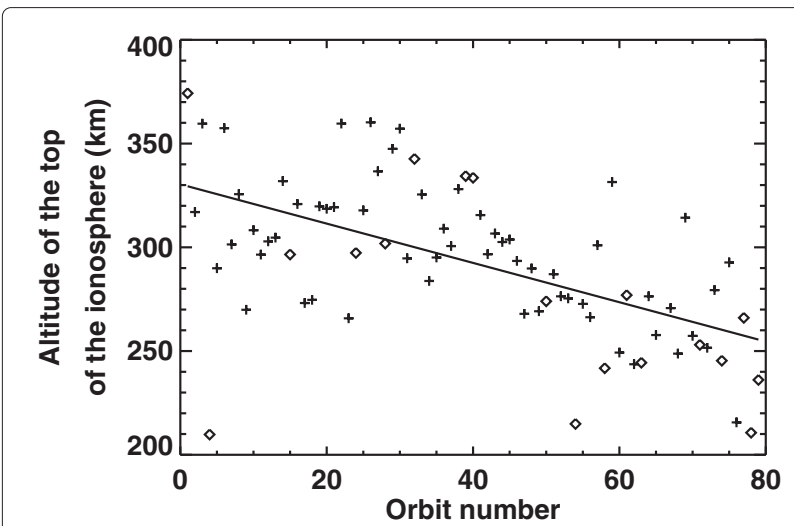

Fig. 5 Altitude of the top of the ionosphere, as defined in the text, as a function of orbit number during the primary mission. Crosses indicate actual detections of the top of the ionosphere, and diamonds indicate lower limits from non-detections. The best fit line is discussed in the text

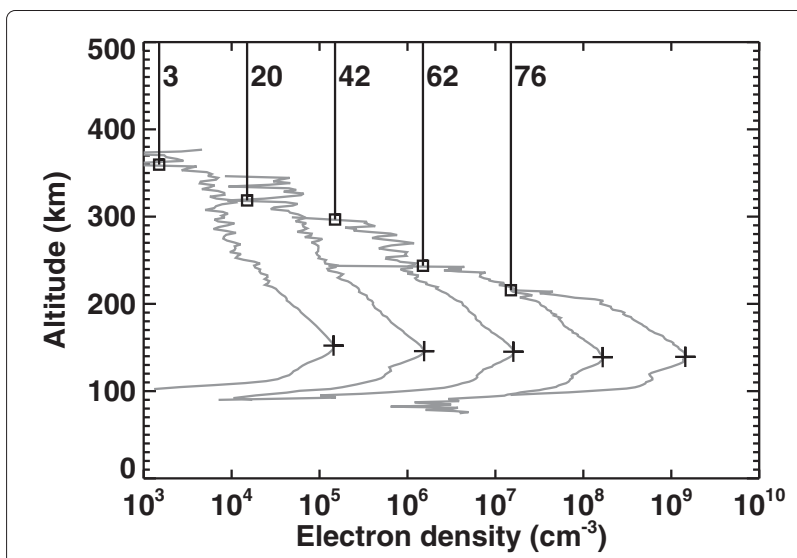

Fig. 6 Five electron density profiles from the primary mission. Orbit numbers are given above each profile. Electron densities in the profiles from orbits 3, 20,42, 62, and 76 have been multiplied by factors of $10^{0}, 10^{1}, 10^{2}, 10^{3}$, and $10^{4}$, respectively, for clarity. Squares at electron densities of $1500 \mathrm{~cm}^{-3}$ indicate the altitude at which electron densities first drop below this value, the top of the ionosphere. Associated vertical lines emphasize the steady decrease in this altitude over time during the primary mission. Crosses indicate the main peak of the ionosphere, which drops in altitude over this period by a much smaller amount

It is hoped that MAVEN studies of the boundary between the ionosphere and the surrounding space environment will shed light on why these differences exist.

\section{Vertical structure of the topside ionosphere}

Withers et al. (2012) investigated the vertical structure of over 500 electron density profiles acquired by MEX radio occultations. In $10 \%$ of the profiles, a single scale height on the order of several tens of kilometers was sufficient to describe the decrease in electron density with increasing altitude in the topside ionosphere. In $25 \%$ of the profiles, two distinct scale heights were necessary, with a scale height on the order of several tens of kilometers changing above a threshold altitude to a scale height on the order of $100 \mathrm{~km}$. A further $10 \%$ of the profiles had three regions with distinct scale heights, while the vertical structure of the topside ionosphere was not readily classifiable in the remaining profiles. A possible interpretation of these observations is that a "one scale height" morphology exists where the vertical transport of plasma to high altitudes is effectively suppressed by horizontal magnetic fields and a "two scale height" morphology exists where it is not. In this scenario, classifications of the morphology of the topside ionosphere reveal information about the magnetic environment and plasma transport.

Previous studies of the Mariner 9 electron density profiles have generally emphasized the presence of a "one scale height" morphology (Breus et al. 1998; Kliore 1992; Ness et al. 2000; Shinagawa 1996; Slavin and Holzer 1982). 
We fit electron densities between 170 and $230 \mathrm{~km}$ in each Mariner 9 profile using an exponential dependence on altitude, then tested whether electron densities at higher altitudes were appreciably greater than those predicted by a simple upwards extension of the fit. If they were, then we labeled the profile as having a "two scale height" morphology. The altitude range of 170 to $230 \mathrm{~km}$ was selected because, as Fig. 7 makes clear, the observed electron density in that range is well represented by an exponential decay. We found that a "one scale height" morphology is five times more prevalent in the Mariner 9 primary mission profiles than a "two scale height" morphology. Six
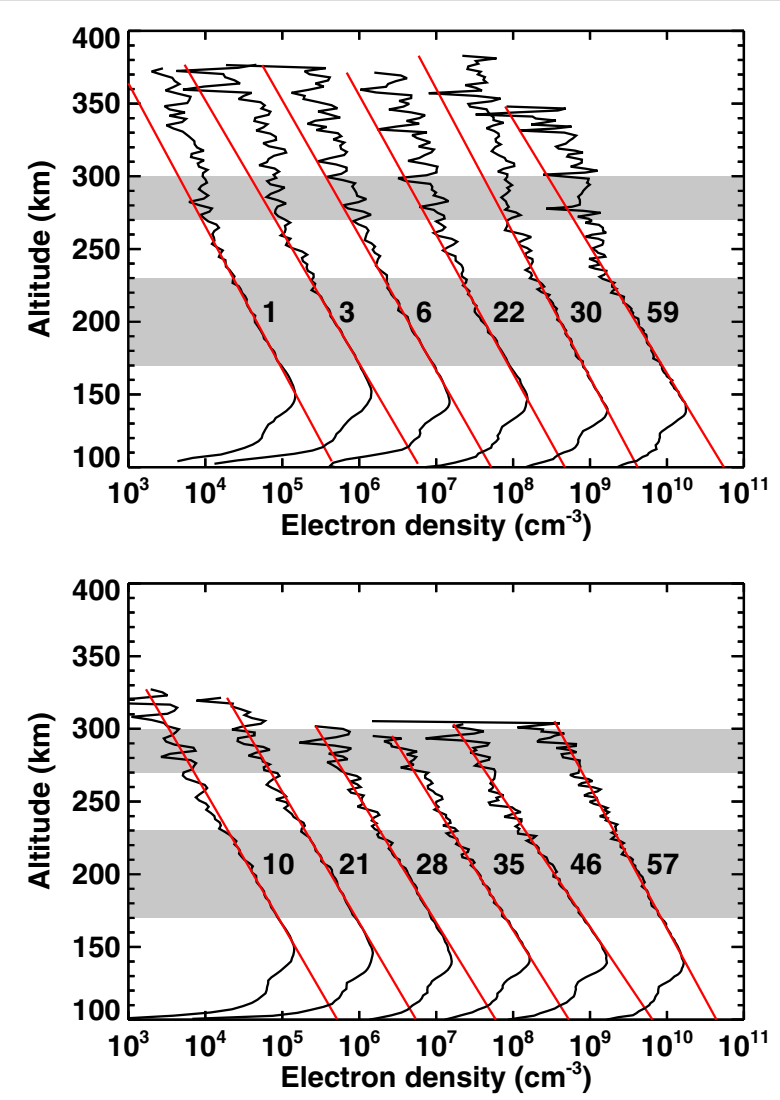

Fig. 7 Top. Six electron density profiles from the primary mission that have a "two scale height" morphology. Orbit numbers are given alongside each profile. Electron densities in the profiles from orbits 1 , $3,6,22,30$, and 59 have been multiplied by factors of $10^{0}, 10^{1}, 10^{2}$, $10^{3}, 10^{4}$, and $10^{5}$ respectively, for clarity. The grey regions highlight 170-230 and 270-300 km altitude. Red lines show extrapolation of fits to data in the 170-230 km altitude range. Observed densities exceed the extrapolation at 270-300 km altitude for these "two scale height" profiles. Bottom. Six electron density profiles from the primary mission that have a "one scale height" morphology. Orbit numbers are given alongside each profile. Electron densities in the profiles from orbits $10,21,28,35,46$, and 57 have been multiplied by factors of $10^{\circ}, 10^{1}$ $10^{2}, 10^{3}, 10^{4}$, and $10^{5}$ respectively, for clarity. Red lines and grey regions as for the top panel, but observed densities do not exceed the extrapolation at 270-300 km altitude for these "one scale height" profiles examples of profiles with a "two scale height" morphology are shown in the top panel of Fig. 7, and six examples of profiles with a "one scale height" morphology are shown in the bottom panel of Fig. 7. The difference in the occurrence rate of these two topside morphologies emphasizes the current inability to predict which morphology will be present in any given observations. In future work, it would be interesting to compare the morphology of the topside ionosphere directly against the strength and direction of the magnetic field.

\section{M1 peak electron density and solar zenith angle}

The dependence of the M1 peak electron density on solar zenith angle has been studied recently by several workers (Fallows et al. 2015; Fox and Yeager 2006; 2009; Liao et al. 2006). However, all used the same dataset from Mars Global Surveyor. It is important to test whether their findings are supported by analysis of an independent dataset. This will also test whether the M1 peak density was disturbed by the dust storm that occurred during the Mariner 9 primary mission.

In order to isolate the M1 layer, we begin with the main peak or M2 layer. Once orbit 4's anomalous result and one result at a solar zenith angle greater than $95^{\circ}$ (orbit $352,98.9^{\circ}$ ) are set aside, the dependence of peak electron density on solar zenith angle appears quite consistent with prior analysis and studies of other datasets (top panel of Fig. 8) (Withers 2009b, and references therein). For instance, a fit of peak density, $N_{m}$, to $C h^{-k}$, where $C h$ is a dimensionless function that reduces to sec SZA for small SZAs and plane-parallel geometry (Chapman 1931a, b; Smith and Smith 1972; Withers 2009b), finds a best-fit subsolar peak density $N_{0}$ of $(2.07 \pm 0.03) \times 10^{5} \mathrm{~cm}^{-3}$ and exponent $k$ of $0.56 \pm 0.01$. The fitted subsolar peak density is also comparable to previous results (Fallows et al. 2015).

Moving to the M1 layer, we fitted the region near the main peak with a Chapman function, which was then subtracted from the observed densities to produce a set of residuals. The residuals in the vicinity of the M1 layer were then fitted with a parabola. The M1 peak altitude was taken to be the fitted altitude of the maximum of the parabola, and the M1 peak density was taken to be the observed density at that altitude. Several profiles from the extended mission did not yield good fits and were not considered further. The fitted M1 peak densities that were deemed acceptable are shown in the bottom panel of Fig. 8. The M1 peak density is proportional to $C h^{-k}$ where $k=0.50 \pm 0.03$. Other workers using electron density profiles from Mars Global Surveyor have found similar exponents (Fallows et al. 2015; Fox and Yeager 2006; 2009; Liao et al. 2006). The fitted subsolar M1 density is also comparable to previous results (Fallows et al. 2015). The result reported here confirms those earlier findings using an independent dataset and also demonstrates that the 

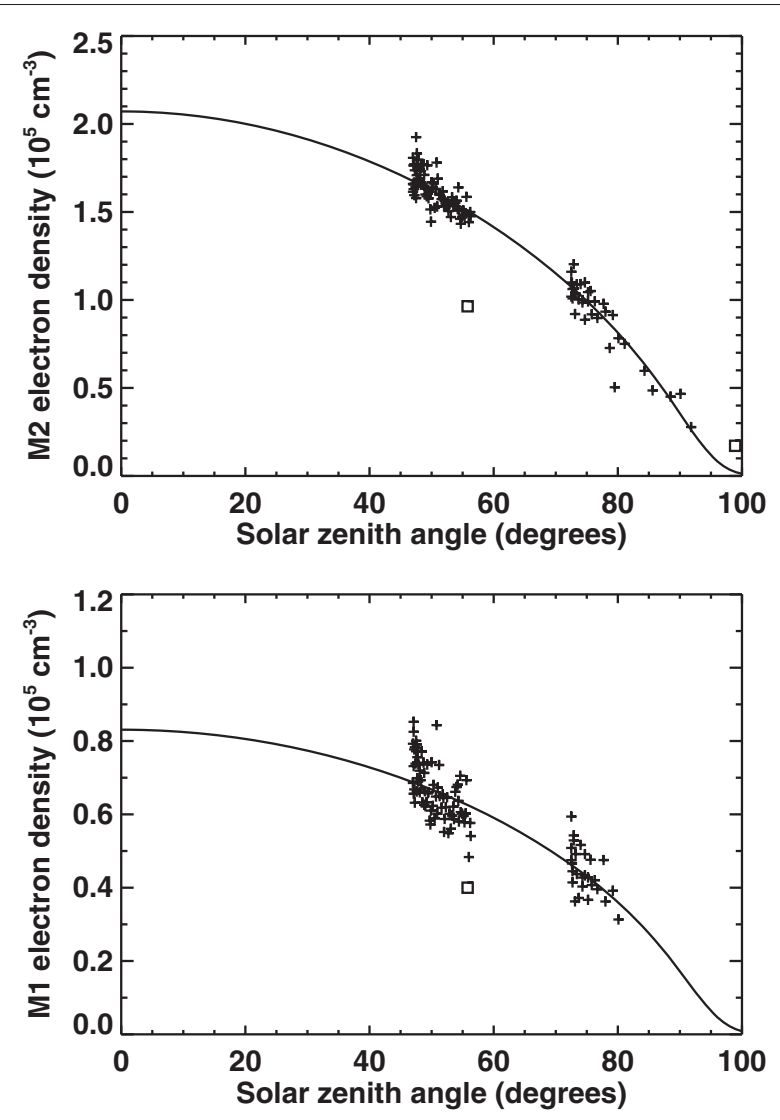

Fig. 8 Top. M2 peak electron density as a function of solar zenith angle. Squares highlight data points excluded from fits. The black line is a fit to data from the primary and extended missions that is discussed further in the text. Bottom. As top panel, but for M1 peak electron densities

Mariner 9 dust storm did not affect M1 peak densities. Previous work found that the dust storm did not affect M2 peak densities (Hantsch and Bauer 1990).

\section{M1 peak electron density and solar irradiance}

Fox and Yeager (2009) investigated the dependence of M1 electron density on solar irradiance using Mars Global Surveyor electron density profiles. Here, we test whether their findings are consistent with analysis of the independent Mariner 9 dataset.

We converted the fitted M1 peak densities obtained in the section "M1 peak electron density and solar zenith angle" to subsolar values using $N_{m}=N_{0} C h^{-0.5}$. For the solar irradiance proxy, we use "adjusted $\mathrm{F}_{10.7}$ " values. $\mathrm{F}_{10.7}$ values at Earth from 1970-1971 were adjusted to values appropriate for use at Mars by time shifting to account for the non-zero Earth-Sun-Mars angle and by multiplying by the inverse square of the Mars-Sun distance. These subsolar M1 peak densities depend on the adjusted value of $F_{10.7}$, as shown in the bottom panel of Fig. 9. They are proportional to the adjusted value of $F_{10.7}$ raised to the
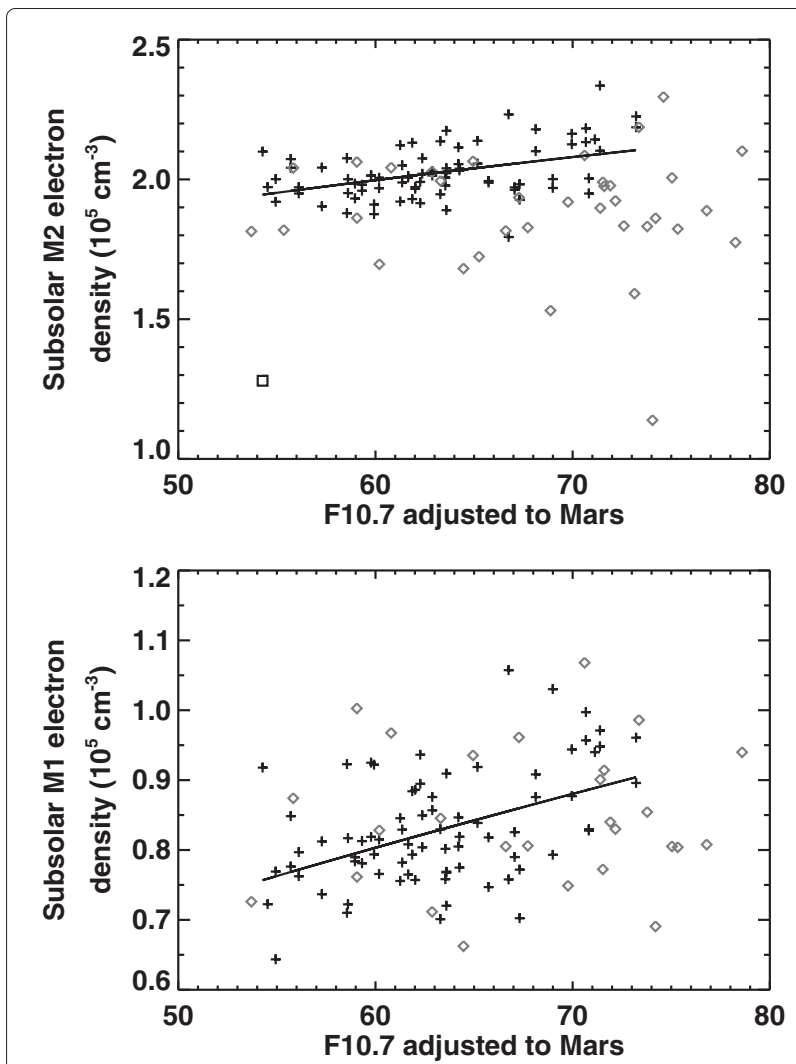

Fig. 9 Top. Inferred subsolar M2 peak electron density as a function of adjusted $F_{10.7}$ (values in solar flux units). Crosses and diamonds indicate occultations from the primary and extended missions, respectively. The solid line is a fit to the primary mission data, excluding the anomalous result from orbit 4 (square symbol). Bottom. As top panel, but for subsolar M1 peak electron density. The anomalous result from orbit 4, which is excluded from the fit, is below the density range of this plot

power $0.59 \pm 0.13$. This fit excludes the extended mission data, since they display much greater scatter than the primary mission data. Nevertheless, inspection of the bottom panel of Fig. 9 shows that the extended mission data are generally consistent with the primary mission data. In their analysis of Mars Global Surveyor data, Fox and Yeager (2009) found an exponent of $0.462 \pm 0.012$.

Due to the large uncertainty on the Mariner 9 exponent, these two exponents are formally indistinguishable. Nevertheless, to explore this topic further, we repeated this analysis for the M2 peak densities and found an exponent of $0.26 \pm 0.06$. Fox and Yeager (2009) reported a very similar value: an exponent of $0.263 \pm 0.008$. There does not appear to be any systematic difference between Mariner 9 and Mars Global Surveyor results for how peak electron densities depend on solar irradiance.

The extended mission M2 peak densities do not have a clear and well-behaved dependence on adjusted $F_{10.7}$ (Fig. 9). The degradation of the experiment between the 
primary and extended missions is one possible explanation, but the well-behaved dependence of peak densities on SZA during the extended mission (the section "M1 peak electron density and solar zenith angle") argues against this. The large Earth-Sun-Mars angle during the extended mission $\left(\sim 130^{\circ}\right.$, in contrast to $\sim 40^{\circ}$ during the primary mission) and the consequent errors in adjusting solar irradiances from Earth to Mars may be responsible.

The difference in exponents between the M1 and M2 layers means that the ratio of M1 to M2 density should increase with increasing solar activity, a natural consequence of the hardening of the solar spectrum. However, due to the relatively small range of adjusted $F_{10.7}$ experienced by Mariner 9, the ratio of the M1 peak density to the M2 peak density remains $\sim 0.4$ in all profiles.

\section{Separation between the $\mathrm{M} 1$ and $\mathrm{M} 2$ layers}

The separation between the M1 and M2 layers was studied by Fallows et al. (2015), who found that it was 25-30 $\mathrm{km}$ and that it did not vary greatly with solar zenith angle. Figure 10 shows the M2 and M1 peak altitudes throughout the course of the mission. The effects of the waning dust storm on the M2 peak altitude have been discussed previously (e.g., Kliore et al. 1973; McElroy et al. 1977; Withers and Pratt 2013). Figure 10 shows that the M1 peak altitude tracks the M2 peak altitude during the storm, being consistently $27 \mathrm{~km}$ lower. With the reasonable assumption that this altitude difference is proportional to the neutral scale height and hence to the neutral temperature, the stability of this altitude difference implies that neutral temperatures at $110-150 \mathrm{~km}$ do not change over the course of the primary mission despite the raging dust storm in the lower atmosphere. The M1 peak altitude is less accurately determined for the extended mission, but values are $\sim 30 \mathrm{~km}$ below the M2 peak on average.

\section{Possible meteoric layers}

The ablation of meteoroids in the upper atmosphere of Mars introduces magnesium, iron, and silicon into the atmosphere. These species modify the behavior of the ionosphere and can produce layers of atomic metal ions around $90 \mathrm{~km}$ (Pätzold et al. 2005; Withers et al. 2008). Based on visual inspection of published figures for layers of plasma near $90 \mathrm{~km}$ altitude, Withers et al. (2013) suggested that several Mariner 9 profiles contained meteoric layers and identified likely meteoric layers in profiles from orbits $5,6,10,30,31,43$, and 67 . The recovery of the Mariner 9 profiles permits critical evaluation of the findings of Withers et al. (2013).

Features suggestive of plasma layers can be seen near $90 \mathrm{~km}$, as shown in Fig. 11, but none of them are reliable. In all cases, the magnitude of the excess plasma density is comparable to the likely uncertainty in the densities, which can be considered equal to the smallest reported density in a given profile. Comparison to the topside strengthens this conclusion: fluctuations in topside density, which are probably caused by instrumental noise, are comparable to the possible meteoric layers at low altitudes. The densities in the possible meteoric layers are roughly the same as those at which fluctuations in topside density become significant. We conclude that no meteoric layers have been firmly identified in the Mariner 9 dataset.

\section{Discussion and conclusions}

The Mariner 9 ionospheric electron density profiles have been recovered from their microfilm archive and have been made widely available by incorporation into this work as Additional file 1. Furthermore, we have recently received a copy of the Viking Orbiter electron density profiles, which are not available on the NASA Planetary Data System, from Tamara Breus (personal communication, 2015). Preparation for documenting them, acquiring the necessary supporting information, and archiving them is underway.

One electron density profile from the Mariner 9 primary mission, orbit 4, is distinctly different from the rest. In this profile, densities in the M1 and M2 layers are tens of percent smaller than in other profiles and the top of the ionosphere is depressed by $60 \mathrm{~km}$. The magnetic conditions at the location of this profile are unlike those experienced
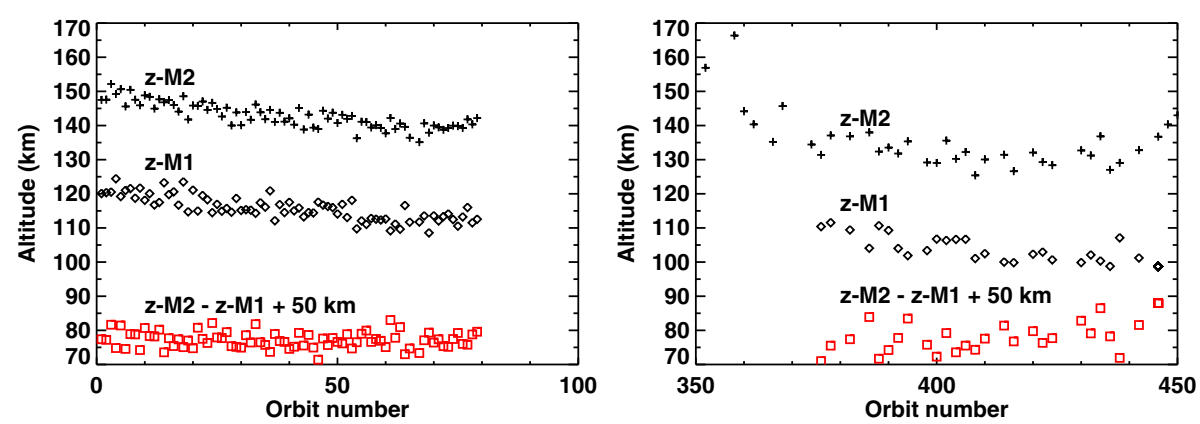

Fig. 10 Peak altitudes of the M2 layer (crosses) and M1 layer (diamonds). The altitude difference is also shown (red squares), though it has been offset by $50 \mathrm{~km}$ for clarity. Left and right panels show occultations from the primary and extended missions, respectively 


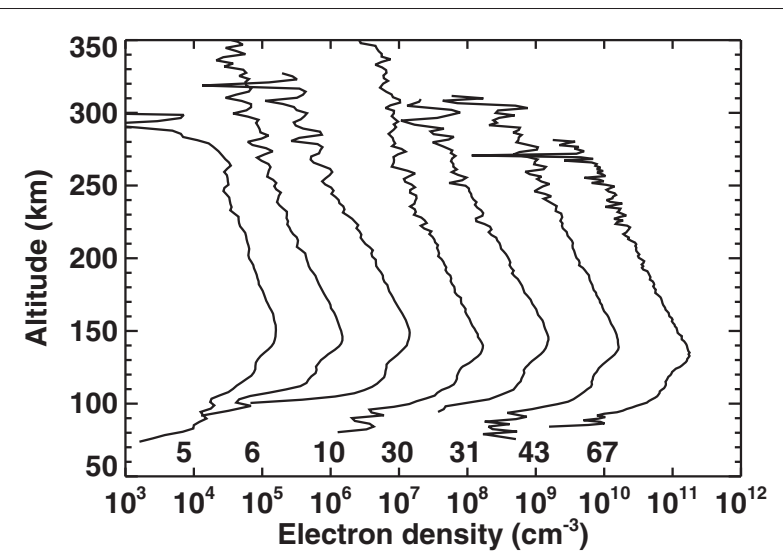

Fig. 11 Seven electron density profiles from the primary mission that have been suggested, based on visual inspection of published figures for layers of plasma near $90 \mathrm{~km}$ altitude, to contain meteoric layers. Orbit numbers are given below each profile. Electron densities in the profiles from orbits $5,6,10,30,31,43$, and 67 have been multiplied by factors of $10^{0}, 10^{1}, 10^{2}, 10^{3}, 10^{4}, 10^{5}$, and $10^{6}$ respectively, for clarity

by the other profiles: the magnetic field is relatively strong and field lines have a relatively high probability of being open. This unusual profile contrasts markedly with the results of Nielsen et al. (2007), who reported cases in which peak densities were approximately doubled where the magnetic field is strong and vertical.

The immense dust storm that waned during the primary mission occultations had major effects on the ionosphere of Mars. It elevated the M2 peak altitude above its nominal level by $20-30 \mathrm{~km}$. It also elevated the M1 peak altitude such that the separation of the M1 and M2 layers stayed fixed throughout the primary mission. The altitude of the top of the ionosphere, defined as the $1500 \mathrm{~cm}^{-3}$ density level, was strongly affected by the dust storm. While the M1 and M2 layers descended by $10 \mathrm{~km}$ over the course of the primary mission as the dust storm waned, the top of the ionosphere descended by $70 \mathrm{~km}$.

The dramatic effects on the topside ionosphere are puzzling. The altitude of the top of the ionosphere could be controlled by solar wind conditions, particularly the dynamic pressure, but it seems unlikely that solar wind conditions would exhibit a secular change over a period of 40 days (the primary mission). It would also be a remarkable coincidence for this unusual behavior in the solar wind to occur at the same time as the greatest dust storm of the last four decades. If this behavior of the topside ionosphere does reflect the effects of a dust storm, then why should a dust storm in the lower atmosphere affect the topside ionosphere so much more than it affects the region around the ionospheric peak? We have no definitive answer to this puzzle and merely note two related recent observations. Liemohn et al. (2012) found that periods of elevated dayside photoelectron flux at $400 \mathrm{~km}$ could be correlated with the average dust opacity over the preceding 7 months. Chaffin et al. (2014) used MEX SPICAM ultraviolet observations to find that the hydrogen escape rate from Mars decreased by an order of magnitude over six months in 2007, coincident with the waning of a large dust storm in fall 2007 (Mars Year 28). This has been corroborated by independent measurements by the Hubble Space Telescope (Bhattacharyya et al. 2014; Clarke et al. 2009).

Moving beyond the effects of the dust storm, there are differences between the topside ionosphere as seen in the Mariner 9/Viking era and as seen in the Mars Express era. First, data from the Mariner 9 extended mission and the Viking Orbiters suggest that electron densities above $250 \mathrm{~km}$ are less than $1500 \mathrm{~cm}^{-3}$, in contrast with Mars Express MARSIS data that suggest that electron densities at $300-350 \mathrm{~km}$ are on the order of $2000-4000 \mathrm{~cm}^{-3}$. Second, the ratio of the occurrence rates of "two scale height" to "one scale height" morphologies is much greater in Mars Express radio occultation data than that in Mariner 9 extended mission data. "Two scale height" morphologies are associated with the ionosphere extending upwards to relatively high altitudes. Both these differences may be caused by relatively strong solar wind dynamic pressures during the Mariner 9 and Viking era by comparison to the Mars Express era (Richardson et al. 2001). Mars Express has operated during a prolonged and deep solar minimum, when solar wind dynamic pressures have been weak. Strong solar wind dynamic pressures depress the top of the ionosphere, which favors a "one scale height" morphology and which also reduces electron densities at high altitudes (Brain 2006; Crider et al. 2003; Mitchell et al. 2001; Opgenoorth et al. 2013). A strong solar wind dynamic pressure is likely to lead to greater penetration of a horizontal magnetic field into the ionosphere, which would suppress the vertical transport of plasma and may therefore favor a "one scale height morphology". In future work, it would be interesting to compare the morphology of the topside ionosphere directly against the strength and direction of the magnetic field.

The M1 layer of the ionosphere has been little-studied to date, with prior analyses having used only a single dataset from Mars Global Surveyor. The dependence of the M1 peak density on solar zenith angle and solar irradiance in the Mariner 9 observations is similar to previous Mars Global Surveyor results, which confirms these earlier results and demonstrates that the intense dust storm during the Mariner 9 primary mission did not affect the M1 peak density appreciably. The separation of the M1 and M2 layers is consistently $25-30 \mathrm{~km}$ in the Mariner 9 dataset, similar to an analysis that used Mars Global Surveyor data. This shows that atmospheric heating due to the dust storm was confined to altitudes below the M1 layer. 


\section{Additional file}

\section{Additional file 1: The 114 Mariner 9 electron density profiles} accompany this article as supplemental information. Each profile is provided in an ASCII text file. A header in the file provides orbit number, date, latitude, longitude, solar zenith angle, radius of reference areoid at that latitude and longitude, radial distance to the maximum electron density in the profile, and the value of the maximum electron density. The body of the file consists of three columns: radial distance, altitude, and electron density. The file naming convention is "orbitXXX.tXt" where "XXX" is the orbit number, which ranges from "001" to "450". (ZIP $269 \mathrm{~kb}$ )

\section{Competing interests}

The authors declare that they have no competing interests.

\section{Authors' contributions}

NF recovered the Mariner 9 data and conducted initial quality control. SW performed the scientific analysis under the direction of PW, who wrote the manuscript. All authors read and approved the final manuscript.

\section{Acknowledgements}

We gratefully acknowledge helpful reviews, Arv Kliore and the team of original investigators for archiving these data, the NSSDC for supplying these data to us, Dave Brain for providing his magnetic field topology maps, Zachary Girazian for producing data products related to the peak properties and solar irradiance, and discussions with Marissa Vogt. This work was supported, in part, by NASA award NNX12AJ39G.

Received: 10 August 2015 Accepted: 27 November 2015 Published online: 03 December 2015

\section{References}

Acuña MH, Connerney JEP, Wasilewski P, Lin RP, Mitchell D, Anderson KA, Carlson CW, McFadden J, Rème H, Mazelle C, Vignes D, Bauer SJ, Cloutier P, Ness NF (2001) Magnetic field of Mars: summary of results from the aerobraking and mapping orbits. J Geophys Res 106:23403-23418

Arkani-Hamed J (2004) A coherent model of the crustal magnetic field of Mars. J Geophys Res 109:E09005. 10.1029/2004JE002265

Bauer SJ, Hantsch MH (1989) Solar cycle variation of the upper atmosphere temperature of Mars. Geophys Res Lett 16:373-376

Bhattacharyya D, Clarke JT, Bertaux J-L, Chaufray J-Y (2014) Analysis and modeling of HST observations of the Martian exosphere. 224th American Astronomical Society Meeting Abstracts, 405.04. http://adsabs.harvard. edu/abs/2015GeoRL..42.8678B

Bougher SW, Engel S, Hinson DP, Forbes JM (2001) Mars Global Surveyor Radio Science electron density profiles: neutral atmosphere implications. Geophys Res Lett 28:3091-3094

Brain DA (2006) Mars Global Surveyor measurements of the martian solar wind interaction. Space Sci Rev 126:77-112

Brain DA, Lillis RJ, Mitchell DL, Halekas JS, Lin RP (2007) Electron pitch angle distributions as indicators of magnetic field topology near Mars. J Geophys Res 112:A09201. 10.1029/2007JA012435

Breus TK, Pimenov KY, Izakov MN, Krymskii AM, Luhmann JG, Kliore AJ (1998) Conditions in the Martian ionosphere/atmosphere from a comparison of a thermospheric model with radio occultation data. Planet Space Sci 46:367-376

Cain DL, Kliore AJ, Seidel BL, Sykes MJ (1972) The shape of Mars from the Mariner 9 occultations. Icarus 17:517-524

Cain DL, Kliore AJ, Seidel BL, Sykes MJ, Woiceshyn P (1973) Approximations to the mean surface of Mars and Mars atmosphere using Mariner 9 occultations. J Geophys Res 78:4352-4354

Chaffin MS, Chaufray J-Y, Stewart I, Montmessin F, Schneider NM, Bertaux J-L (2014) Unexpected variability of Martian hydrogen escape. Geophys Res Lett 41:314-320

Chapman S (1931a) The absorption and dissociative or ionizing effect of monochromatic radiation in an atmosphere on a rotating Earth. Proc Phys Soc 43:26-45
Chapman, S (1931b) The absorption and dissociative or ionizing effect of monochromatic radiation in an atmosphere on a rotating Earth. Part II. Grazing incidence. Proc Phys Soc 43:483-501

Clarke JT, Bertaux J, Chaufray J, Gladstone R, Quemerais E, Wilson JK (2009) HST observations of the extended hydrogen corona of Mars. Paper presented at the 41 st American Astronomical Society Division for Planetary Sciences Meeting, Abstract 49.11, Fajardo, Puerto Rico, 4-9 October 2009

Crider DH, Vignes D, Krymskii AM, Breus TK, Ness NF, Mitchell DL, Slavin JA, Acuñ MH (2003) A proxy for determining solar wind dynamic pressure at Mars using Mars Global Surveyor data. J Geophys Res 108:1461. 10.1029/2003JA009875

Duru F, Gurnett DA, Morgan DD, Modolo R, Nagy AF, Najib D (2008) Electron densities in the upper ionosphere of Mars from the excitation of electron plasma oscillations. J Geophys Res 113:A07302. 10.1029/ 2008JA013073

Fallows K, Withers P, Matta M (2015) An observational study of the influence of solar zenith angle on properties of the M1 layer of the Mars ionosphere. J Geophys Res 120:1299-1310

Fjeldbo G, Sweetnam D, Brenkle J, Christensen E, Farless D, Mehta J, Seidel B, Michael W, Wallio A, Grossi M (1977) Viking radio occultation measurements of the Martian atmosphere and topography — primary mission coverage. J Geophys Res 82:4317-4324

Fox JL, Yeager KE (2006) Morphology of the near-terminator martian ionosphere: a comparison of models and data. J Geophys Res 111:A10309. 10.1029/2006JA011697

Fox JL, Yeager KE (2009) MGS electron density profiles: analysis of the peak magnitudes. Icarus 200:468-479

Hantsch MH, Bauer SJ (1990) Solar control of the Mars ionosphere. Planet Space Sci 38:539-542

Hinson DP (2007) MGS RST Science Data Products, USA_NASA_JPL_MORS_1102. In: Simpson RA (ed). MGS-M-RSS-5-EDS-V1.0. NASA Planetary Data System

Kliore A, Cain DL, Siedel BL, Fjeldbo G (1970) S-band occultation for Mariner Mars 1971. Icarus 12:82-90

Kliore AJ (1973) Radio occultation exploration of Mars(Woszczyk A, Iwaniszewska C, eds.), Vol. 65. Exploration of the Planetary System, IAU Proceedings

Kliore, A J (1992) Radio occultation observations of the ionospheres of Mars and Venus. In: Venus and Mars: Atmospheres, ionospheres, and solar wind interactions. Vol 66 of Geophysical Monograph Series. American Geophysical Union. DC, Washington. pp 265-276

Kliore AJ, Cain DL, Fjeldbo G, Seidel BL, Rasool SI (1972a) Mariner 9 S-band martian occultation experiment: initial results on the atmosphere and topography of Mars. Science 175:313-317

Kliore AJ, Cain DL, Fjeldbo G, Seidel BL, Sykes MJ, Rasool SI (1972b) The atmosphere of Mars from Mariner 9 radio occultation measurements. Icarus 17:484-516

Kliore AJ, Fjeldbo G, Seidel BL, Sykes MJ, Woiceshyn PM (1973) S band radio occultation measurements of the atmosphere and topography of Mars with Mariner 9: extended mission coverage of polar and intermediate latitudes. J Geophys Res 78:4331-4351

Liao H-R, Wang J-S, Zou H, Wang X-D (2006) Observational features of the secondary layer of the martian ionosphere. In: Bhardwaj A (ed). Advances in Geosciences: Volume 3. World Scientific Publishing, Singapore. pp 135-143. http://adsabs.harvard.edu/abs/2006aogs....3..135L

Liemohn MW, Dupre A, Bougher SW, Trantham M, Mitchell DL, Smith MD (2012) Time-history influence of global dust storms on the upper atmosphere at Mars. Geophys Res Lett 39. 10.1029/2012GL051994, L11201

Luhmann JG (1992) Comparative studies of the solar wind interaction with weakly magnetized planets. Adv Space Res 12:191-203

Luhmann JG, Brace LH (1991) Near-Mars space. Rev Geophys 29:121-140

Luhmann JG, Kliore A, Barnes A, Brace L (1990) Remote sensing of Mars' ionosphere and solar wind interaction-lessons from Venus. Adv Space Res 10:43-48

McElroy MB, Kong TY, Yung YL (1977) Photochemistry and evolution of Mars' atmosphere-a viking perspective. J Geophys Res 82:4379-4388

Mitchell DL, Lin RP, Rème H, Cloutier PA, Connerney JE, Acuña MH, Ness NF (2001) Probing Mars' crustal magnetic field with the MGS electron reflectometer. Paper presented at the AGU Spring Meeting, Abstract GP22A-7, Boston, 29 May - 2 June 2001 
Mitchell DL, Lin RP, Rè H, Cloutier PA, Connerney JE, Acuñ MH, Ness NF (2001) Probing Mars' crustal magnetic field with the MGS electron reflectometer. AGU Spring Meeting abstract GP22A-07

Ness NF, Acuña MH, Connerney JEP, Kliore AJ, Breus TK, Krymskii AM, Cloutier P, Bauer SJ (2000) Effects of magnetic anomalies discovered at Mars on the structure of the martian ionosphere and solar wind interaction as follows from radio occultation experiments. J Geophys Res 105:15991-16004

Nielsen E, Fraenz M, Zou H, Wang J-S, Gurnett DA, Kirchner DL, Morgan DD, Huff R, Safaeinili A, Plaut JJ, Picardi G, Winningham JD, Frahm RA, Lundin R (2007) Local plasma processes and enhanced electron densities in the lower ionosphere in magnetic cusp regions on Mars. Planet Space Sci 55:2164-2172

Opgenoorth HJ, Andrews DJ, Frä M, Lester M, Edberg NJT, Morgan D, Duru F, Witasse O, Williams AO (2013) Mars ionospheric response to solar wind variability. J Geophys Res 118:6558-6587

Pätzold M, Tellmann S, Häusler B, Hinson D, Schaa R, Tyler GL (2005) A sporadic third layer in the ionosphere of Mars. Science 310:837-839

Richardson JD, Wang C, Paularena KI (2001) The solar wind: from solar minimum to solar maximum. Adv Space Res 27:471-479

Schunk RW, Nagy AF (1980) lonospheres of the terrestrial planets. Rev Geophys Space Phys 18:813-852

Shinagawa H (1996) Our current understanding of the ionosphere of Mars. Adv Space Res 26:1599-1608

Slavin JA, Holzer RE (1982) The solar wind interaction with Mars revisited. J Geophys Res 87:10285-10296

Smith DE, Zuber MT, Frey HV, Garvin JB, Head JW, Muhleman DO, Pettengill GH, Phillips RJ, Solomon SC, Zwally HJ, Banerdt WB, Duxbury TC, Golombek MP, Lemoine FG, Neumann GA, Rowlands DD, Aharonson O, Ford PG, Ivanov AB, Johnson CL, McGovern PJ, Abshire JB, Afzal RS, Sun X (2001) Mars Orbiter Laser Altimeter: experiment summary after the first year of global mapping of Mars. J Geophys Res 106:23689-23722

Smith FL, Smith C (1972) Numerical evaluation of Chapman's grazing incidence integral Ch(X, х). J Geophys Res 77:3592-3597

Stewart AJ, Hanson WB (1982) Mars' upper atmosphere-mean and variations. Adv Space Res 2:87-101

Wang J-S, Nielsen E (2003) Behavior of the Martian dayside electron density peak during global dust storms. Planet Space Sci 51:329-338

Withers P (2009a) A review of observed variability in the dayside ionosphere of Mars. Adv Space Res 44:277-307

Withers, P (2009b) A review of observed variability in the dayside ionosphere of Mars. Adv Space Res 44:277-307

Withers P, Christou AA, Vaubaillon J (2013) Meteoric ion layers in the ionospheres of Venus and Mars: early observations and consideration of the role of meteor showers. Adv Space Res 52:1207-1216

Withers P, Fallows K, Girazian Z, Matta M, Häusler B, Hinson D, Tyler L, Morgan D, Pätzold M, Peter K, Tellmann S, Peralta J, Witasse O (2012) A clear view of the multifaceted dayside ionosphere of Mars. Geophys Res Lett 39:L18202. 10.1029/2012GL053193

Withers P, Mendillo M, Hinson DP, Cahoy K (2008) Physical characteristics and occurrence rates of meteoric plasma layers detected in the martian ionosphere by the Mars Global Surveyor Radio Science Experiment. J Geophys Res 113:A12314. 110.1029/2008JA013636

Withers P, Pratt R (2013) An observational study of the response of the upper atmosphere of Mars to lower atmospheric dust storms. Icarus 225:378-389

Zhang MHG, Luhmann JG (1992) Comparisons of peak ionosphere pressures at Mars and Venus with incident solar wind dynamic pressure. J Geophys Res 97:1017-1025

Zhang MHG, Luhmann JG, Kliore AJ, Kim J (1990) A post-Pioneer Venus reassessment of the martian dayside ionosphere as observed by radio occultation methods. J Geophys Res 95:14829-14839

\section{Submit your manuscript to a SpringerOpen ${ }^{\circ}$ journal and benefit from:}

- Convenient online submission

- Rigorous peer review

- Immediate publication on acceptance

Open access: articles freely available online

- High visibility within the field

- Retaining the copyright to your article

Submit your next manuscript at springeropen.com 